
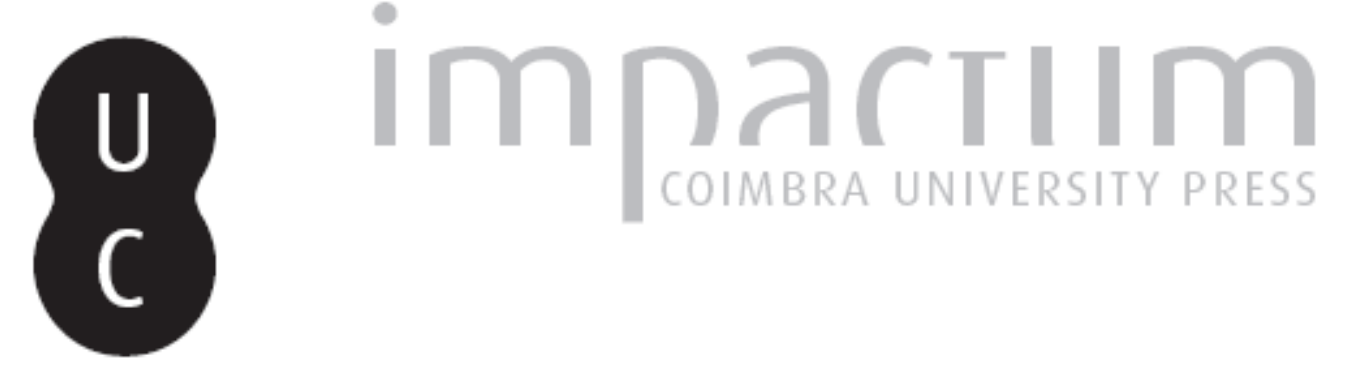

\title{
Mitigação do risco sísmico de núcleos urbanos antigos: caracterização e avaliação da vulnerabilidade sísmica do Bairro Ribeirinho de Faro
}

Autor(es): $\quad$ Vicente, Romeu; Ferreira, Tiago M.; Maio, Rui

Publicado por: $\begin{aligned} & \text { Associação Portuguesa de Riscos, Prevenção e Segurança; Imprensa } \\ & \text { da Universidade de Coimbra }\end{aligned}$

URL

persistente:

DOI: $\quad$ DOl:http://dx.doi.org/10.14195/1647-7723_22_22

Accessed : $\quad$ 26-Apr-2023 12:54:23

A navegação consulta e descarregamento dos títulos inseridos nas Bibliotecas Digitais UC Digitalis, UC Pombalina e UC Impactum, pressupõem a aceitação plena e sem reservas dos Termos e Condições de Uso destas Bibliotecas Digitais, disponíveis em https://digitalis.uc.pt/pt-pt/termos.

Conforme exposto nos referidos Termos e Condições de Uso, o descarregamento de títulos de acesso restrito requer uma licença válida de autorização devendo o utilizador aceder ao(s) documento(s) a partir de um endereço de IP da instituição detentora da supramencionada licença.

Ao utilizador é apenas permitido o descarregamento para uso pessoal, pelo que o emprego do(s) título(s) descarregado(s) para outro fim, designadamente comercial, carece de autorização do respetivo autor ou editor da obra.

Na medida em que todas as obras da UC Digitalis se encontram protegidas pelo Código do Direito de Autor e Direitos Conexos e demais legislação aplicável, toda a cópia, parcial ou total, deste documento, nos casos em que é legalmente admitida, deverá conter ou fazer-se acompanhar por este aviso.

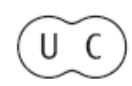




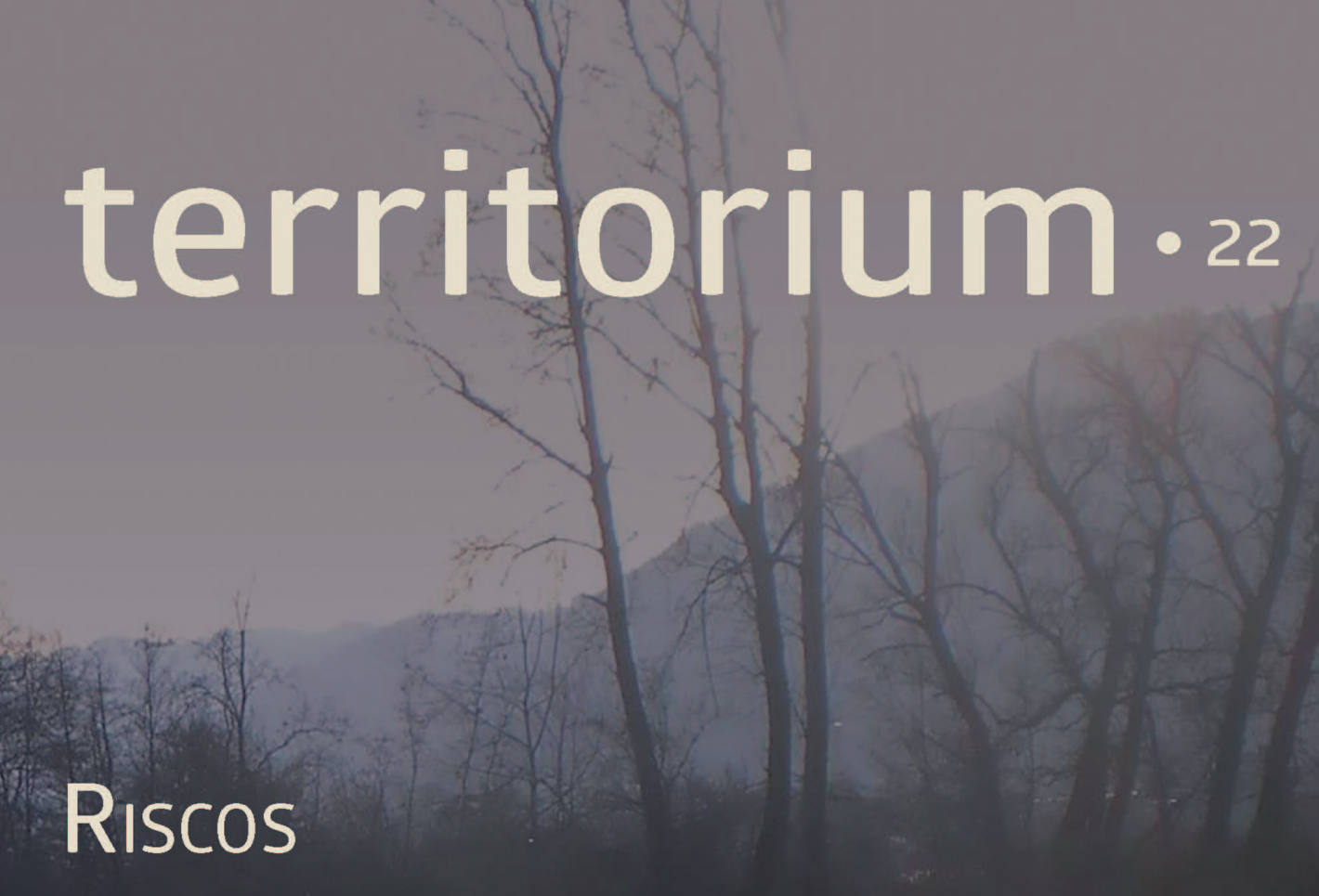

\section{TERRITÓRIOS DE CONVERGÊNCIA}

- Imprensa da Universidade de Coimbra Associação Portuguesa de:Riscos, Prevenção e Segurança 


\section{MITIGAÇÃO DO RISCO SÍSMICO DE NÚCLEOS URBANOS ANTIGOS: CARACTERIZAÇÃO E AVALIAÇÃO DA VULNERABILIDADE SÍSMICA DO BAIRRO RIBEIRINHO DE FARO*}

\section{SEISMIC RISK MITIGATION OF OLD URBAN CENTRES: CHARACTERIZATION AND SEISMIC VULNERABILITY ASSESSMENT OF THE RIBEIRINHA NEIGHBORHOOD OF FARO, PORTUGAL}

Romeu Vicente

RISCO, Departamento de Engenharia Civil, Universidade de Aveiro romvic@ua.pt

Tiago M. Ferreira

RISCO, Departamento de Engenharia Civil, Universidade de Aveiro tmferreira@ua.pt

Rui Maio

RISCO, Departamento de Engenharia Civil, Universidade de Aveiro ruiamaio@ua.pt

\section{RESUMO}

A vulnerabilidade sísmica do Bairro Ribeirinho de Faro é avaliada neste artigo através da aplicação de uma metodologia simplificada, a partir da qual foram construídos diferentes cenários de dano físico e estimadas perdas económicas e humanas. Os resultados apresentados confirmam um cenário bastante desfavorável, mas que poderá servir de base à implementação de medidas preventivas por parte das entidades decisoras, no sentido de minorar a vulnerabilidade e incrementar a resiliência deste núcleo urbano antigo.

Palavras-chave: Risco sísmico, vulnerabilidade sísmica, núcleos urbanos antigos, cenários de dano e perdas.

\section{ABSTRACT}

The seismic vulnerability of the old city centre of Faro is assessed in this paper resorting to a simplified assessment methodology from which different scenarios of physical damage and losses were obtained. Such results confirm a very unfavorable scenario but that can be used by governmental authorities and civil protection bodies to put into practice preventive mitigation measures in order to reduce vulnerability and improve the site/local resilience.

Keywords: Seismic risk, seismic vulnerability, old urban centres, damage and loss scenarios.

\section{Introdução}

O património edificado nos centros históricos das nossas cidades é denunciador de um vasto número de indicadores culturais, sociais e económicos, que possibilitam entender a sua morfologia e traçar o seu processo evolutivo, desde a sua origem até aos dias de hoje. Devido ao efeito combinado entre o avançado estado atual de degradação de uma parte significativa do edificado urbano antigo e o facto de se tratarem de construções seculares, edificadas antes da entrada em vigor de qualquer regulamentação sísmica, a vulnerabilidade sísmica nestas áreas é manifestamente elevada, justificando por isso uma atenção redobrada. Sendo a perigosidade sísmica do território Português moderada a elevada, o risco sísmico e de tsunami é uma ameaça real e que deve ser minimizado de forma proactiva através de uma estreita colaboração entre todas as entidades intervenientes neste processo.

Perante este quadro, o presente artigo aborda o estudo da vulnerabilidade sísmica do edificado como a medida mais eficaz na mitigação do risco sísmico de núcleos urbanos antigos. Dada a incerteza associada à ocorrência de um evento, a perigosidade sísmica é, de entre os três vetores definidores de risco sísmico, aquele que não é passível de ser minimizado. A exposição, a qual avalia o valor dos elementos em risco (humanos e materiais), poderá contribuir para a mitigação do risco sísmico através da adopção de medidas que visem condicionar a construção e o desenvolvimento das cidades em locais de elevada perigosidade sísmica e de descentralizar e distribuir

* O texto desta nota corresponde a uma comunicação apresentada no III Congresso Internacional, I Simpósio Ibero-Americano e VIII Encontro Nacional de Riscos, tendo sido submetida em 02-02-2015, sujeita a revisão por pares a 30-04-2015 e aceite para publicação em 23-07-2015.

Esta nota é parte integrante da Revista Territorium, $n .{ }^{\circ} 22,2015,{ }^{\circ}$ RIscos, ISSN: 0872-8941. 
as grandes massas humanas e centros económicos e financeiros destas áreas de risco. Com base no exposto, a redução da vulnerabilidade sísmica do edificado urbano apresenta-se como a estratégia mais imediata na mitigação do risco sísmico. Assim sendo, e porque a implementação de qualquer estratégia de redução da vulnerabilidade deverá assentar impreterivelmente no conhecimento da condição dessa mesma vulnerabilidade, este artigo apresenta o processo de construção de cenários de perda (patrimonial, humana e económica), baseados na aplicação de uma metodologia simplificada de avaliação da vulnerabilidade sísmica. Esta metodologia, adaptada ao caso Português por Vicente (2008) é baseada num índice de vulnerabilidade estimado a partir de informação qualitativa e quantitativa relacionada com as caraterísticas geométricas e propriedades mecânicas do edificado.

Refira-se ainda que, de forma a melhorar e optimizar a gestão das características do parque edificado, foi desenvolvido e utilizado um sistema de informação geográfica em software ArcGis $10.2^{\circledR}$ (ESRI, 2011), o qual combina dados georreferenciados com informação relativa a cada edifício avaliado.

\section{O centro histórico de Faro como caso de estudo}

Tal como ilustrado na fig. 1, o centro histórico de Faro encontra-se subdivido em três núcleos edificados, associados às áreas de reabilitação urbana delimitadas pela Câmara Municipal de Faro: Vila Adentro (Zona A), Mouraria (Zona B) e Bairro Ribeirinho (Zona C), este último utilizado neste trabalho como zona de estudo para a avaliação da vulnerabilidade sísmica através da já mencionada metodologia de proposta por Vicente et al. (2011), aqui apresentada de forma resumida na Secção 2.
A seleção do Bairro Ribeirinho de Faro como caso de estudo baseou-se no facto de esta ser uma das zonas com maior concentração de edifícios de alvenaria de pedra, muitos destes com níveis de alteração estrutural reduzidos, premissa essencial para a aplicação da referida metodologia.

o levantamento e caracterização tipológica e construtiva do património edificado do Bairro Ribeirinho, que antecedeu naturalmente o presente trabalho de avaliação da vulnerabilidade sísmica, revelou-se uma tarefa fundamental para um melhor conhecimento estrutural das tipologias existentes naquele núcleo edificado, tornando assim mais célere o processo de avaliação da vulnerabilidade sísmica segundo a metodologia utilizada. De um modo geral, e no que ao tipo de paredes resistentes diz respeito, foram observados os seguintes sistemas: construção mista em taipa composta ou por fragmentos de pedra argamassados com areia, terra e cal (fig. 2 (d)), ou por uma alvenaria em blocos de adobe; construção em alvenaria de pedra de fraca qualidade (de origem aluvionar) com a presença de detritos cerâmicos (ver fig. 2 (a)), construção mista em taipa de melhor qualidade, com cantaria e cunhais em pedra calcária, e por último, construção porticadas em betão armado com painéis de enchimento em alvenaria de tijolo, esta última fora do contexto da nossa avaliação.

Não menos importante, as coberturas, que assumem um papel crucial na preservação geral do edifício e que acabam muitas vezes por determinar o estado de conservação dos restantes elementos estruturais e não estruturais. No Bairro Ribeirinho de Faro foram encontradas diversas tipologias, desde os tradicionais "telhados em tesouro" (coberturas inclinadas) na fig. 2 (b), aos terraços em "Santa Catarina”

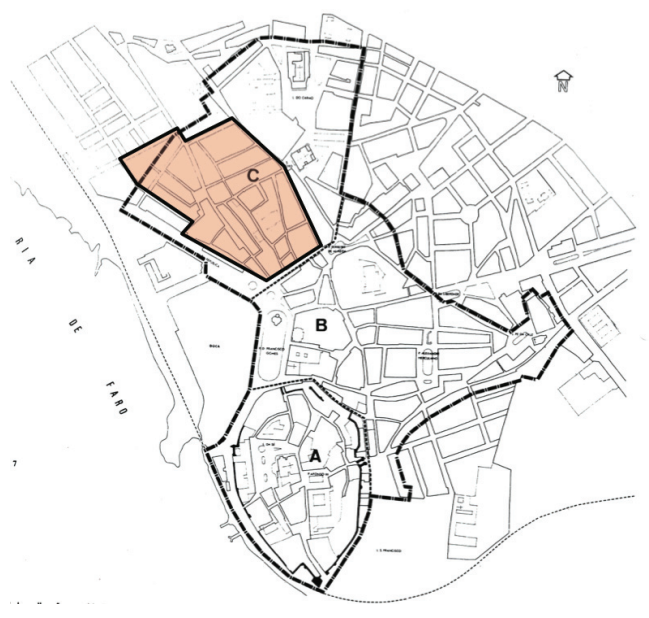

(a)

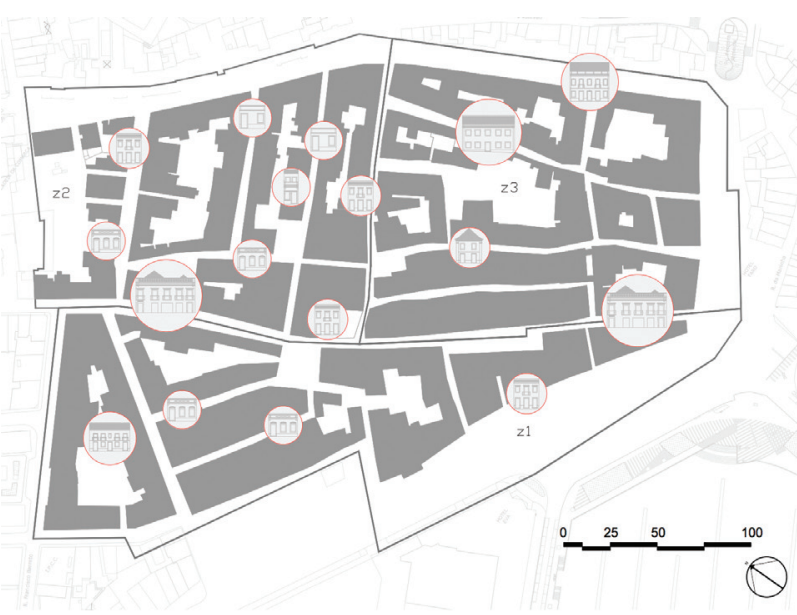

(b)

Fig. 1 - Localização do Bairro Ribeirinho (Zona C) no núcleo urbano antigo da cidade de Faro (a) e o levantamento e caracterização tipológica (b).

Fig. 1 - Location of the Bairro Ribeirinho (Zone C) within the old city centre of Faro (a) and its respective typological classification. 
(coberturas planas) na fig. 2 (e). Por último, no que diz respeito aos diafragmas horizontais, foi observada a presença de arcos e abóbadas de alvenaria, com ou sem vigamento metálico (ver fig. 2 (f)), vestígio da utilização das semicaves de alguns destes edifícios como antigos armazéns. Quanto aos pavimentos, a sua estrutura tradicional era normalmente realizada em vigamentos (barrotes) de madeira encastrados diretamente nas paredes resistentes ou simplesmente apoiados por arcos, abóbadas ou abobadilhas, constituídas por elementos cerâmicos ou pétreos (fig. 2 (c)). 0 soalho e os ladrilhos cerâmicos cozidos são os dois tipos de revestimento mais frequentemente observados no edificado antigo do Bairro Ribeirinho de Faro.

A necessidade de adaptação do tipo de utilização dos edifícios em função da evolução da morfologia da cidade de Faro potenciou a grande maioria das intervenções e alterações no edificado do Bairro Ribeirinho. Os grandes armazéns foram divididos em lotes mais pequenos, dando lugar a habitações unifamiliares, ao aproveitamento dos pisos térreos para a instalação de comércio, contribuindo definitivamente para a zona do Bairro Ribeirinho de Faro se tenha afirmado como um dos polos mais importantes de atração noturna na cidade. 0 ruído gerado por estes estabelecimentos, particularmente durante o período noturno, num Bairro de cariz marcadamente habitacional, tem motivado frequentes queixas dos residentes, situação que contribui para a elevada taxa de edifícios devolutos e em ruína. Simultaneamente, esta situação potencia o desinteresse de possíveis investidores dispostos a apostar na reabilitação de alguns destes edifícios.

Destaque ainda para a existência de inúmeros edifícios em situação de ruína ou pré-ruína, e para as intervenções de adaptação e reabilitação de edifícios realizadas ao longo das últimas duas décadas, as quais foram responsáveis pela descaracterização de algum do edificado, levando à perda de uma parte importante deste património arquitectónico. Refiram-se ainda algumas alterações que, pela sua natureza, podem apresentar consequência estruturais graves, tais como a abertura de grandes vãos na parede de fachada ou a supressão de elementos resistentes ao nível do rés- dochão, situações tipicamente decorrentes da mudança de função do edifício. Note-se que as alterações de uso ou de ocupação de um edifício são aceitáveis, desde que não motivem intervenções estruturais e arquitectónicas intrusivas e que sejam devidamente pautadas pelo rigor e ponderação (T. M. Ferreira et al., 2013a).

Finalmente importa ainda destacar a falta de coerência arquitectónica da zona de estudo, situação claramente recorrente da fiscalização desadequada das intervenções de ampliação destes edifícios, com resultado numa sobreposição desadequada, por vezes labiríntica, de volumes cujas consequências em termos de resposta estrutural são é muitas vezes desconhecida.

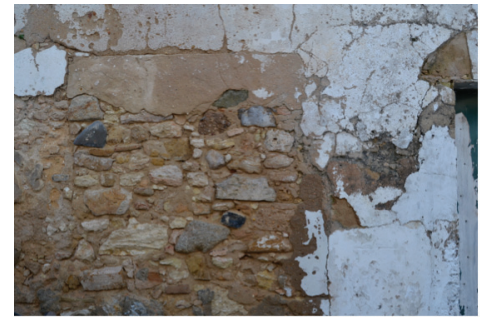

(a)

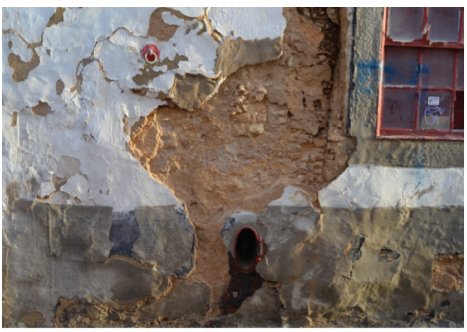

(d)

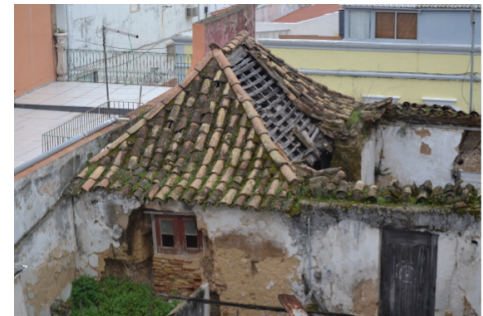

(b)

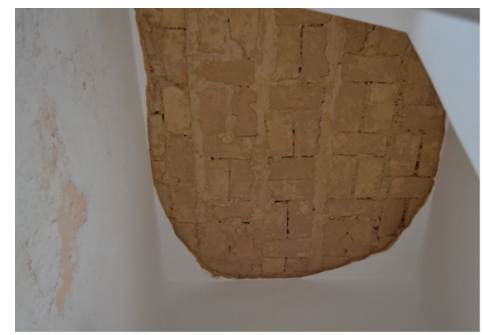

(e)

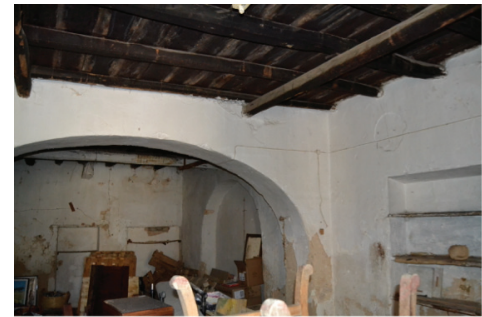

(c)

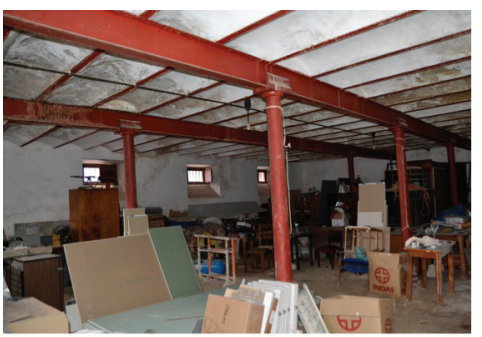

(f)

Fig. 2 - Exemplo de alvenaria de pedra muito pobre e irregular (a) e paredes em taipa com pequenos fragmentos de pedra (d), "telhados em tesouro" (b) e terraços em "Santa Catarina" (e), e ainda, estrutura de pavimento com barrotes de madeira (c) e com vigamento metálico (f).

Fig. 2 - Few examples of local stone masonry with poor and irregular fabric (a) "taipa" traditional constructive typology with small stone fragments (d), "tesouro" traditional structural roofing system (b) and "Santa Catarina" terraces (e), and finally traditional timber (c) and steel (f) flooring systems. 


\section{Metodologia do Índice de Vulnerabilidade}

A formulação do índice de vulnerabilidade sísmica utilizada neste trabalho, apresentada de forma resumida no QUADRo I, foi originalmente desenvolvida em Itália pelo GNDT II (1994) para a avaliação da vulnerabilidade sísmica de edifícios de alvenaria através da observação e catalogação de danos e mecanismos de colapso após a ocorrência de um evento sísmico. Desde a sua origem, este método tem sido amplamente utilizado por diversos autores (Giovinazzi and Lagomarsino (2004); Calvi and Pinho (2006); Guéguen et al. (2007); Srikanth et al. (2010); Athmani et al. (2015), etc.). Em Portugal, esta metodologia foi adaptada à realidade dos nossos edifícios antigos por Vicente (2008), introduzindo uma análise e inspeção mais cuidada e ainda outros novos parâmetros contabilizando a interação entre edifícios adjacentes. Inicialmente aplicada à Baixa da cidade de Coimbra (Vicente et al., 2011), esta metodologia foi recentemente utilizada na avaliação do núcleo urbano antigo do Seixal (T. M. Ferreira et al., 2013b).

Sucintamente, a sua aplicação consiste na determinação de um índice de vulnerabilidade, $I_{v}{ }^{*}$, o qual é calculado para cada edifício através de uma média ponderada de 14 parâmetros distribuídos em 4 classes de vulnerabilidade crescente, $C_{v i}$, de $A$ a $D$. A cada parâmetro é ainda associado um determinado peso, $p_{i}$, definido em função da relevância desse parâmetro para o comportamento sísmico do edifício.

$$
I_{v}^{*}=\sum_{i=1}^{14} C_{v i} \times p_{i}
$$

Este índice é posteriormente normalizado, para variar entre 0 e 100, assumindo a designação final de $I_{v}$. A definição detalhada de cada um destes 14 parâmetros pode ser consultada em Vicente (2008).

Note-se que a principal fonte de incerteza associada ao método reside na definição dos pesos, $p_{i}$, associados a cada um dos parâmetros utilizados. A este respeito, Vicente (2008) refere que caso a inspeção do edifício a avaliar seja realizada com o detalhe necessário e exista informação geométrica suficiente e rigorosa, este método pode ser considerado robusto e a incerteza associada à seleção das classes de vulnerabilidade é relativamente baixa.

\section{Resultados}

Dadas as dificuldades encontradas durante o período de inspeção e levantamento dos edifícios pertencentes ao núcleo urbano antigo do Bairro Ribeirinho, foram

QUADRo I - Formulação da metodologia do índice de vulnerabilidade em Vicente (2008).

TABLE I - Formulation of the seismic vulnerability index methodology by Vicente (2008).

\begin{tabular}{|c|c|c|c|c|c|c|c|}
\hline \multirow{2}{*}{\multicolumn{2}{|c|}{ Parâmetros }} & \multicolumn{4}{|c|}{ Classe $C_{v i}$} & \multirow{2}{*}{$\frac{\text { Peso }}{p_{i}}$} & \multirow{2}{*}{$\begin{array}{c}\text { Peso } \\
\text { relativo }\end{array}$} \\
\hline & & A & $\mathrm{B}$ & $\mathrm{C}$ & $D$ & & \\
\hline \multicolumn{8}{|c|}{ Grupo 1. Sistema construtivo } \\
\hline P1 & Tipo de sistema resistente & 0 & 5 & 20 & 50 & 0,75 & \multirow{6}{*}{$46 / 100$} \\
\hline P2 & Qualidade do sistema resistente & 0 & 5 & 20 & 50 & 1,00 & \\
\hline P3 & Resistência convencional & 0 & 5 & 20 & 50 & 1,50 & \\
\hline P4 & Distância máxima entre paredes & 0 & 5 & 20 & 50 & 0,50 & \\
\hline P5 & Número de pisos & 0 & 5 & 20 & 50 & 1.50 & \\
\hline P6 & Fundações e tipo de solo & 0 & 5 & 20 & 50 & 0.75 & \\
\hline
\end{tabular}

Grupo 2. Irregularidades e interações

\begin{tabular}{llllllll}
\hline P7 & Posição e interação no agregado & 0 & 5 & 20 & 50 & 1,50 & $27 / 100$ \\
P8 & Configuração em planta & 0 & 5 & 20 & 50 & 0,75 & 0.75 \\
P9 & Regularidade em altura & 0 & 5 & 20 & 50 & \\
\hline \multicolumn{7}{c}{ Grupo 3. Pavimentos e cobertura } \\
\hline \multirow{2}{*}{ P10 } & Alinhamento de aberturas & 0 & 5 & 20 & 50 & 0,50 & $15 / 100$ \\
P11 & Diafragmas horizontais & 0 & 5 & 20 & 50 & 1,00 & 1,00 \\
P12 & Sistema de cobertura & 0 & 5 & 20 & 50 & \\
\hline
\end{tabular}

Grupo 4. Estado de conservação e outros elementos

$\begin{array}{lllllll}\text { P13 } & \text { Patologias e estado de conservação } & 0 & 5 & 20 & 50 & 1,00 \\ \text { P14 } & \text { Elementos não estruturais } & 0 & 5 & 20 & 50 & 0,50\end{array}$


considerados na nossa análise, diferentes níveis de detalhe. Assim os 354 edifícios que compõe o Bairro Ribeirinho foram divididos em três grandes grupos. 0 primeiro, composto por 53 edifícios para os quais foi possível realizar uma inspeção detalhada, foi avaliado através do preenchimento de cinco fichas de inspeção adaptadas de trabalhos anteriores realizados pela mesma equipa (Ferreira et al., 2013b). O segundo, agrupou cerca de 138 edifícios, para os quais apenas foi possível efetuar uma inspeção pelo exterior (nãodetalhada). Finalmente, foi ainda considerado um terceiro grupo, composto por 163 edifícios, que agrupou os edifícios que devido aos seus materiais, propriedades e sistemas construtivos (edifícios de betão armado), estado de conservação atual (reabilitados ou em ruína) ou estado de ocupação (devolutos) não foram avaliados neste trabalho.

A aplicação da metodologia do índice de vulnerabilidade a cada um dos 191 edifícios avaliados é apresentada na fig. 3 (a), através do mapeamento do $I_{v}$ para cada edifício. Já na fig. 3 (b) são identificados os edifícios com um valor de $I_{v}$ superior a 40 , e que deverão por isso, ser motivo de maior atenção e preocupação no estabelecimento de prioridades e estratégias de intervenção. Note-se que aproximadamente 15\% dos edifícios apresentam um $I_{v}$ superior a 40 , com $5 \%$ acima de 45 , o equivalente a uma classe de vulnerabilidade A na escala EMS-98 (G. Grünthal, 1998). Por outro lado, apenas $4 \%$ dos edifícios avaliados apresentam um $I_{v}$ inferiores a 20 (equivalente a uma classe de vulnerabilidade B na mesma escala EMS-98).

Relativamente aos valores médios do índice de vulnerabilidade, $I_{v \text { médio }}$, foi efetuada uma primeira avaliação considerando apenas os 53 edifícios avaliados detalhadamente para a qual foi obtido um $I_{v, \text { médio }}$ igual a 36.15 .
De acordo com esta metodologia, o grau de dano médio, $\mu_{D}$, pode ser estimado para diferentes intensidades através da aplicação das seguintes equações, desenvolvidas para esse fim por Bernardini et al. (2007):

$$
\begin{gathered}
\mu_{\mathrm{D}}=2.5 \times\left[1+\tanh \left(\frac{1+6.25 \times V-13.1}{Q}\right)\right] ; 0 \leq \mu_{\mathrm{D}} \leq 5 \\
V=0.592+0.0057 \times I_{\mathrm{v}}
\end{gathered}
$$

onde I representa a perigosidade sísmica descrita em termos de intensidade macrossísmica, $V$ representa o índice de vulnerabilidade (calculado na equação anterior), e $Q$ é um factor de ductilidade definido em função da tipologia construtiva do edifício a avaliar (considerado neste trabalho igual a 3.0). A fig. 4 (a) e (b) apresenta os cenários de grau de dano médio obtidos para intensidade macrossísmicas, $I_{\text {(EMS-98) }}$, VIII e IX, respetivamente.

A estimativa de edifícios colapsados e inutilizáveis, resultado de extrema importância e utilidade para as entidades governamentais e de proteção civil, foi neste artigo avaliada com base num modelo desenvolvido por Bramerini et al. (1995), que define os estados de dano que relacionam a probabilidade de se exceder um determinado grau de dano com a probabilidade de colapso e perda funcional. A estimativa do número total de edifícios colapsados e inutilizáveis para diferentes intensidades sísmicas, $I_{\text {(EMS-98) }}$, e para o valor $I_{v, \text { médio }}=34.12$, encontra-se apresentada na fig. 5 .

Também na avaliação das perdas humanas e número de desalojados, foi utilizado o modelo desenvolvido por Bramerini et al. (1995). Para tal, definiu-se a percentagem de mortos e feridos graves como sendo $30 \%$ do número total de habitantes residentes em edifícios colapsados

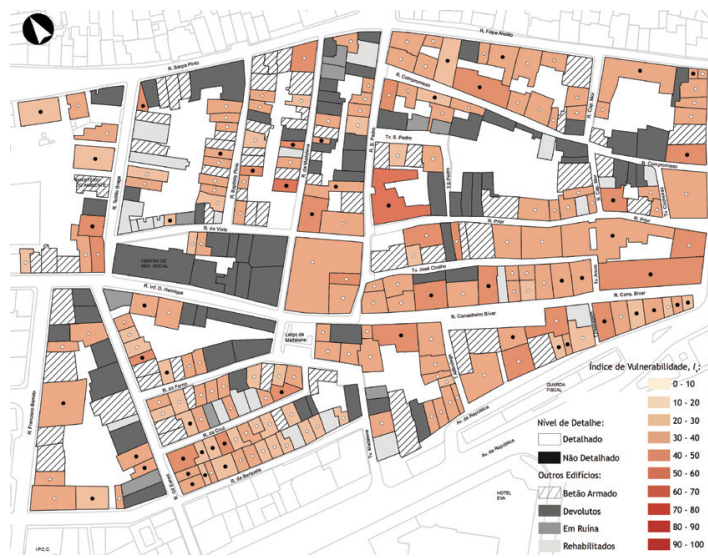

(a)

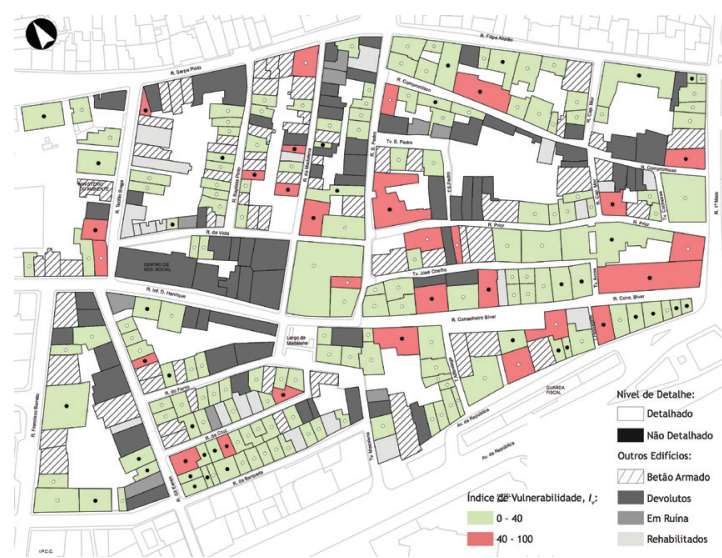

(b)

Fig. 3 - Mapeamento do índice de vulnerabilidade $I_{v}$ para o Bairro Ribeirinho de Faro (a) e identificação dos edifícios com Iv $>40$ (b).

Fig. 3 - Mapping of the vulnerability índex $I_{v}$ for the Rairro Ribeirinho of Faro (a) and the identification of the buildings with a vulnerability index over $I v>40$ (b). 


\section{(}

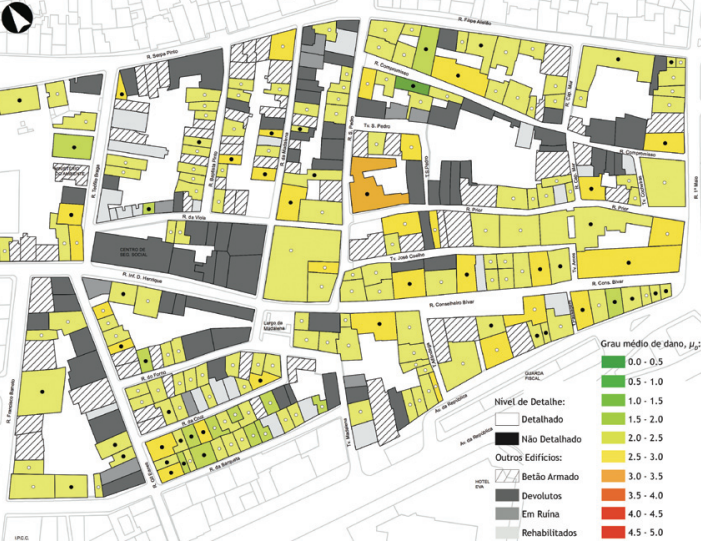

(a)

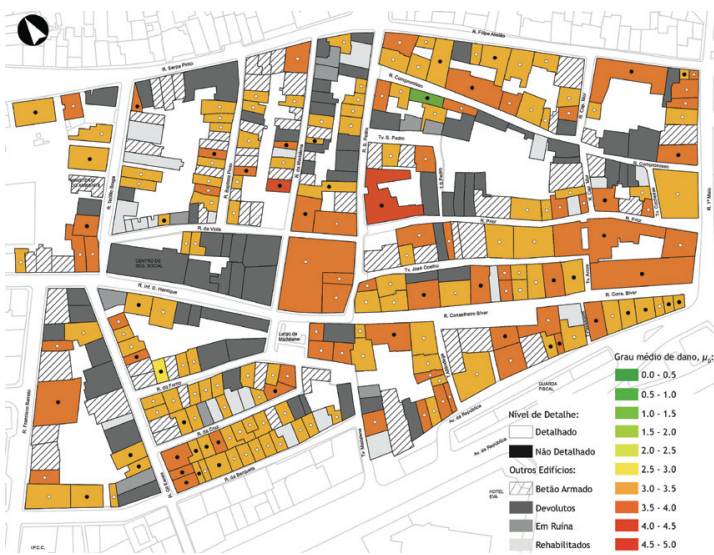

(b)

Fig. 4 - Cenários de dano para intensidades $I_{\text {(ENS-98) }}=$ VIII (a) e $I_{(E M S-98)}=I X(b)$.

Fig. 4 - Damage scenarios for macroseismic intensities $I_{(E M S-98)}=V I I I(a)$ and $I_{(E M S-98)}=I X(b)$.

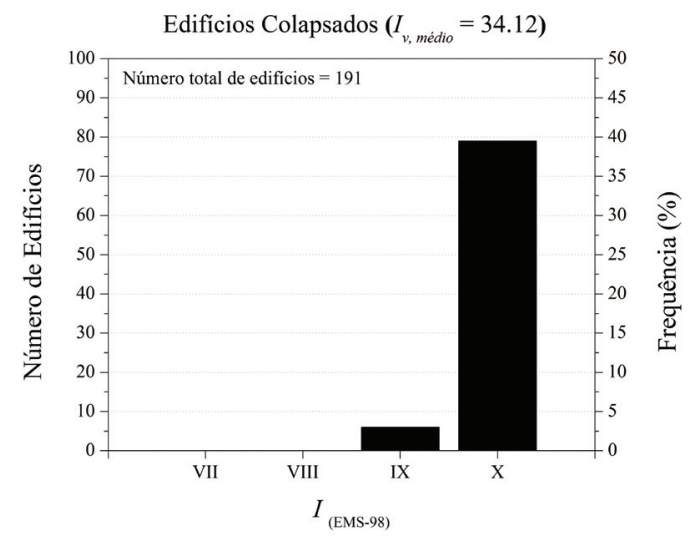

(a)

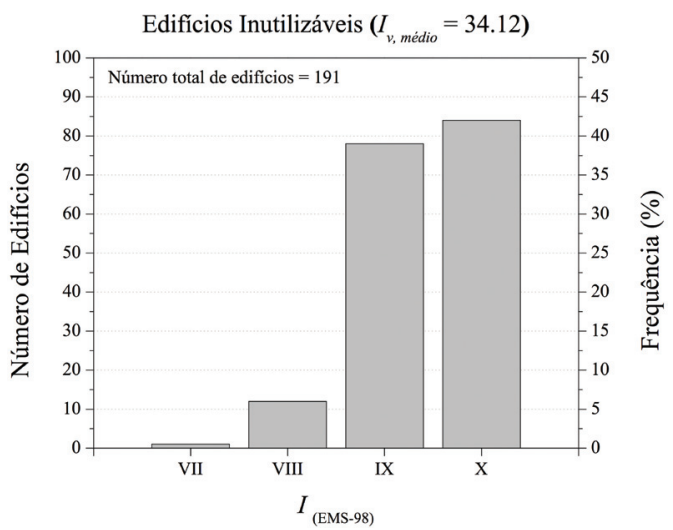

(b)

Fig. 5 - Estimativa do número de edifícios colapsados (a) e inutilizáveis (b).

Fig. 5 - Estimation of the number of collapsed (a) and unusable (b) buldings.

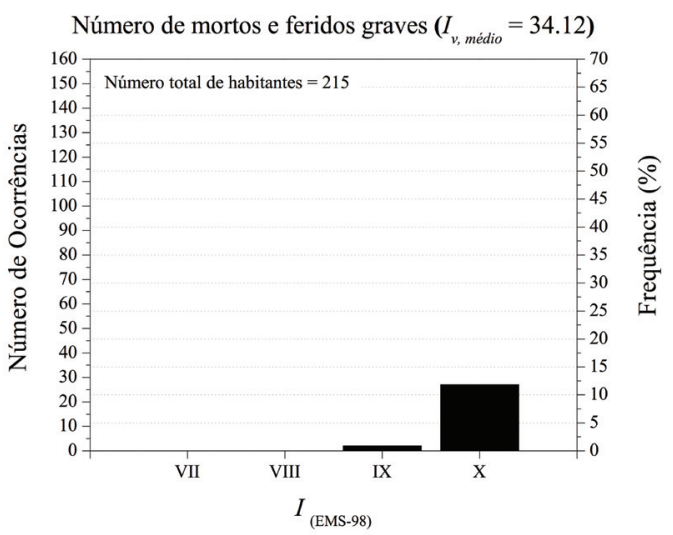

(a)

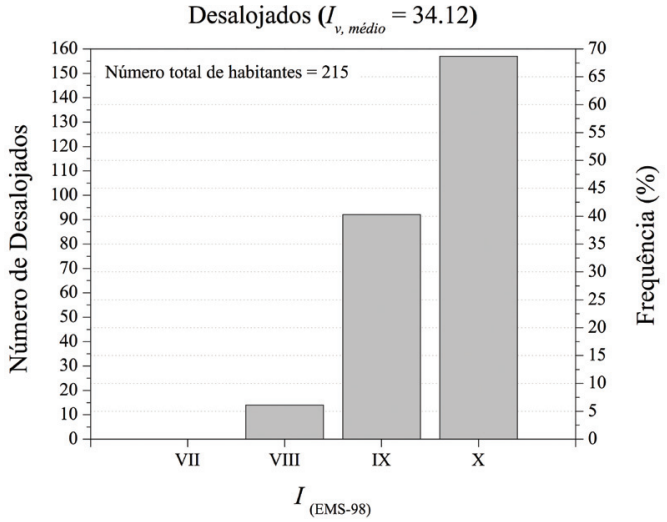

(b)

Fig. 6 - Estimativa do número de mortos e feridos graves (a) e desalojados (b).

Fig. 6 - Estimation of the number of deaths and and severely injured (a) and homeless people (b). 
e inutilizáveis. A estimativa do número de mortos e feridos graves e de desalojados, para o $I_{v, \text { mean }}=34.12$, é apresentada na fig. 6. Importa no entanto salientar que estes números podem ser significativamente agravados em função da sazonalidade própria da região do Algarve e da hora a que ocorra o sismo.

Finalmente, de acordo com Vicente et al. (2011) a probabilidade de custo de reparação para um determinado evento sísmico caracterizado por uma intensidade $I, P[R \mid I]$, pode ser calculada através do produto da probabilidade condicional do custo de reparação para cada grau de dano, $P\left[R \mid D_{k}\right]$ com a probabilidade condicional de condição de dano para cada nível de vulnerabilidade e intensidade sísmica, $P\left[D_{k} \mid I_{v}, I\right]$. Assim, de forma a estimar o custo de reparação associado a diferentes valores de vulnerabilidade na estimativa de perdas, considerou-se um custo unitário de reparação de $482 € / \mathrm{m}^{2}$ em função do valor sugerido pela Portaria n³70/2013 (Portugal, 2013). Com base neste dado, a fig. 7 apresenta a estimativa global dos custos de reparação para o Bairro Ribeirinho de Faro.

\section{Conclusões}

É fundamental que a legislação que regula a reabilitação de edifícios antigos de valor patrimonial reconhecido se estenda para além da conservação da fachada, criando condições e eventuais contrapartidas para os interessados em preservar e reabilitar com as técnicas e soluções construtivas originais. As leis e a burocracia associada ao licenciamento e execução das obras de reabilitação devem ser aligeiradas, de forma a tornar este processo mais célere, simples e económico, atraindo desta forma potenciais investidores. Parece claro que existem ainda situações em que as políticas e as estratégias atuais de incentivo à reabilitação urbana não conseguem dar uma resposta eficiente. No entanto, este descongestionamento processual deve ser acompanhado de uma estratégia de fiscalização mais rígida e penalizadora para os incumpridores, com o intuito de evitar a intrusividade e consequente descaracterização arquitectónica do património edificado. Por outro lado, sublinhe-se que uma avaliação rigorosa e competente da vulnerabilidade sísmica dos núcleos históricos antigos, complementada com soluções apropriadas de reforço estrutural e sísmico, podem reduzir significativamente os danos e as perdas humanas e económicas causados por futuros eventos. Neste sentido, é fundamental promover a reabilitação estrutural e sísmica do património edificado, com base em projetos de reabilitação concebidos de acordo com os princípios definidos pelo ICOMOS (2003).

Assim, de um modo geral os resultados obtidos nesta avaliação podem dizer-se coerentes com as características e o estado de conservação do edificado do Bairro Ribeirinho de Faro. Como nota final refira-se ainda que o valor médio obtido para o índice de vulnerabilidade sísmica deste edificado, igual a 34.14 , encontra-se em linha com os valores obtidos pelos autores em outros estudos realizados no passado, nomeadamente para o edificado antigo da Baixa de Coimbra $\left(I_{v \text {,médio }}=38.38\right)$ e do núcleo urbano antigo do Seixal $\left(I_{v, \text { médio }}=32.81\right)$. No entanto, sendo a perigosidade sísmica da cidade de Faro moderada a elevada, estes resultados revelam que a ocorrência de evento sísmico de intensidade moderada poderá causar danos e perdas significativas, motivadas, quer pela vulnerabilidade do próprio edificado, quer pela falta de preparação das entidades responsáveis e sensibilização da população. Resultado idêntico foi ainda apresentado em 2010 pela Autoridade Nacional de Proteção Civil (ANPC), num estudo sobre o risco sísmico e de tsunamis na região do Algarve (ANPC, 2010).

\section{Agradecimentos}

Este trabalho foi financiado pela Fundação para a Ciência e a Tecnologia (FCT) ao abrigo do projeto de investigação URBSIS: Avaliação da Vulnerabilidade e Gestão do Risco Sísmico à Escala Urbana (PTDC/ECM-URB/2564/2012).

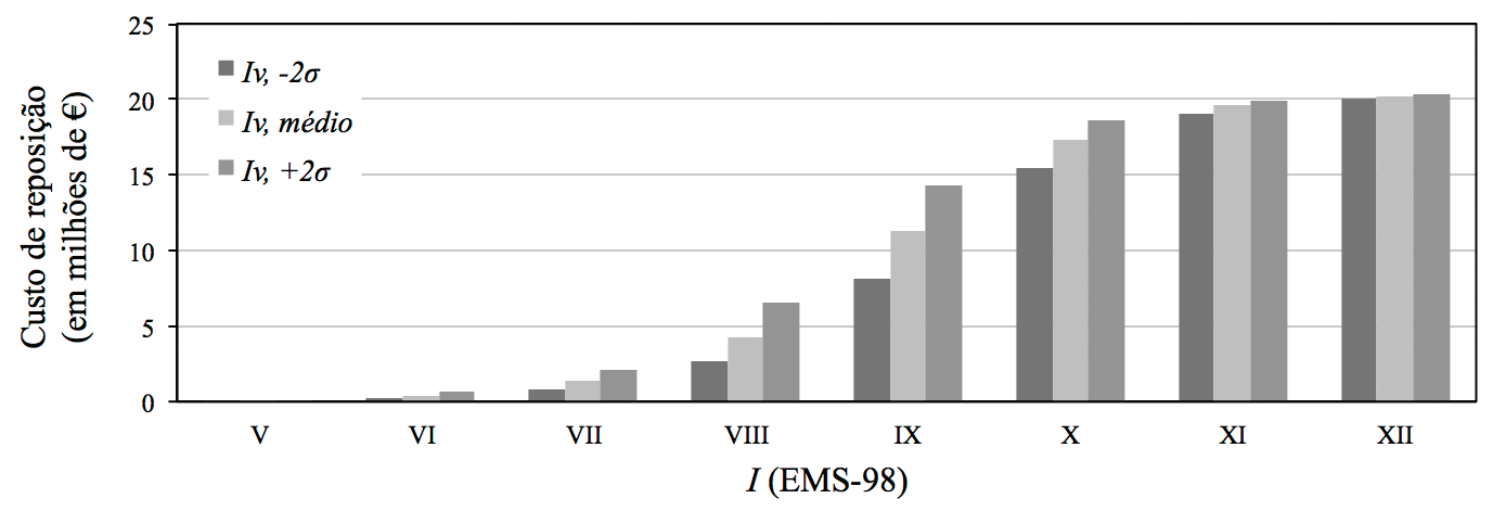

Fig. 7 - Estimativa dos custos de reparação do Bairro Ribeirinho de Faro, para diferentes intensidades $\mathrm{I}_{(\mathrm{EMS}-98)}$.

Fig. 7 - Estimation of the repair costs associated with the Bairro Ribeirinho of Faro, for different macroseismic intensities. 


\section{Bibliografia}

ANPC - AUTORIDADE NACIONAL DE PROTECÇ̃̃O CIVIL (2010). Estudo do Risco Sísmico e de Tsunamis do Algarve. Edição da Autoridade Nacional de Proteção Civil. Coordenação da Direção Nacional de Planeamento de Emergência. Dezembro de 2010. ISBN: 978-989-8343-06-2

Athmani, A.E., Gouasmia, A., Ferreira, T.M., Romeu Vicente, R., Khemis, A. (2015). Seismic vulnerability assessment of historical masonry buildings located in Annaba city (Algeria) using non ad-hoc data survey. Bulletin of Earthquake Engineering. Springer.

Doi:10.1007/s10518-014-9717-7

Bernardini, A., Giovinazzi, S., Lagomarsino, S. e Parodi, S. (2007). Vulnerabilità e previsione di danno a scala territoriale secondo una metodologia macrosismica coerente con la scala EMS-98". ANIDIS, In: XII Convegno Nazionale l'ingegneria sismica in Italia. 10-14 Junho, Pisa, Itália.

Bramerini, F., Di Pasquale, G., Orsini, A., Pugliese, A., Romeo, R. e Sabetta, F. (1995). Rischio sismico del territorio italiano: proposta per una metodologia e risultati preliminar. Servizio Sismico Nazionale, Relatório Técnico SSN/ RT/95/01, Roma.

Calvi GM, Pinho R (2006). Development of seismic vulnerability assessment methodologies over the past 30 years. ISET Journal of Earthquake Technology, Vol. 43(3):75-104.

ESRI (2011). Geographic Information Systems. Nova lorque $n .^{\circ} 310$, Redlands, 92373-8100, E.U.A.

Ferreira, T. M., Santos, C., Vicente, R., Mendes da Silva, J. A. R. (2013a). Caracterização arquitectónica e construtiva do património edificado do núcleo urbano antigo do Seixal. Conservar Património, n. ${ }^{\circ} 17,2013$, p. 21-37, português, DOI:10.14568/cp2012008

Ferreira, T. M., Vicente, R., Mendes da Silva, J. A. R., Varum, H. e Costa, A. (2013b). Seismic vulnerability assessment of historical urban centres: case study of the old city centre in Seixal, Portugal. Bulletin of Earthquake Engineering, Springer, vol. 11, p. 1753-1773, DOI:10.1007/s10518-013-9447-2
Giovinazzi S, Lagomarsino S (2004). A macroseismic method for the vulnerability assessment of buildings. In: $13^{\text {th }}$ World Conference on Earthquake Engineering, Vancouver, BC. Canada.

GNDT (1994). Scheda di esposizione e vulnerabilità e di rilevamento danni di primo e secondo livello (murata e cemento armato). Gruppo Nazionale per la Difesa dai Terremoti, Roma, Itália.

Grünthal, G. (1998). European Macroseismic Scale 1998 (EMS-98), European Seismological Commission, Subcommission on Engineering Seismology, Working Group Macroseismic Scales, 15:101, Luxemburgo.

Gueéguen P, Michel C, LeCorre L (2007). A simplified approach for vulnerability assessment in moderate-to- low seismic hazard regions: application to Grenoble (France). Bulletin of Earthquake Engineering, Springer, Holanda, vol. 5(3):467-490, Doi:10.1007/s10518-007-9036-3

ICOMOS (2003). ICOMOS - Recomendações para a análise, conservação e restauro estrutural do património arquitectónico, 2003.

Portugal (2013). Portaria n०370/2013, de 27 de Dezembro de 2013. Ministério do Ambiente e do Ordenamento do Território, Diário da República, $1^{\text {a }}$ série, $n^{\circ} 251$, Portugal.

Srikanth T, Kumar RP, Singh AP, Rastogi BK, Kumar S (2010). Earthquake vulnerability assessment of existing buildings in Gandhidham and Adipur Cities, Kachchh, Gujarat (India). European Journal of Scientific Research, 41(3):336-353.

Vicente, R. S. (2008). Estratégias e metodologias para intervenções de reabilitação urbana. Avaliação da vulnerabilidade e do rísco sísmico do edificado da Baixa de Coimbra (Tese de Doutoramento). Universidade de Aveiro, Portugal.

Vicente, R. S., Parodi, S., Lagomarsino, S., Varum, H. (2011). Seismic vulnerability and risk assessment: case study of the historic city centre of Coimbra, Portugal. Bulletin of Earthquake Engineering, Springer, Holanda, vol. 9(4), p. 1067-1096. 